\title{
Optimization of oven drying conditions for lycopene content and lipophilic antioxidant capacity in a by-product of the pink guava puree industry using response surface methodology.
}

\begin{abstract}
Response surface methodology (RSM) was applied to optimize the oven drying conditions for lycopene content (Y1) and lipophilic antioxidant capacity (Y2) in decanter, a by-product of the pink guava puree industry. Two-factor central composite design was employed to determine the effects of two independent variables, namely temperature (X1: 50-80 ${ }^{\circ} \mathrm{C}$ ) and drying time (X2: 4-6 h). Lycopene content and lipophilic antioxidant capacity were measured using high-performance liquid chromatography (HPLC) and the ABTS radicals scavenging assay, respectively. A $\beta$-carotene bleaching assay was also applied to measure the antioxidant activity. Response surface plots showed that an increase in temperature and time significantly reduced the response variables. The optimum oven conditions for drying of decanter with minimum lycopene degradation were $43.8{ }^{\circ} \mathrm{C}$ for $6.4 \mathrm{~h}$, with a predicted lycopene content of $14 \mathrm{mg} / 100 \mathrm{~g}$ and antioxidant capacity of $21 \mu \mathrm{mol} \mathrm{LE} / 100 \mathrm{~g}$. To validate the optimized model, the experimental values were compared with the predicted values to check the adequacy of the model. The experimental values were found to be in agreement with those predicted, indicating the suitability of the model for optimizing the oven drying conditions for decanter.
\end{abstract}

Keyword: Oven drying; Lycopene; Antioxidant capacity;Psidium guajava by-products; Response surface methodology 